\title{
Correspondence
}

\section{Genetic background and tumour susceptibility in mouse models}

\author{
Cell Death and Differentiation (2013) 20, 964; doi:10.1038/cdd.2013.35; published online 26 April 2013
}

\section{Dear Editor,}

Genetically engineered mouse (GEM) models of tumourigenesis have revolutionised our understanding of the molecular mechanisms of cancer. They have been instrumental in the identification and characterisation of oncogenes and tumour suppressors and have provided invaluable tools for recapitulating human cancers. However, a major caveat of using GEM models is the phenotypic variation that arises due to the influence of genetic background. ${ }^{1,2}$ Even after extensive backcrossing (>10 times), genes physically linked to the deleted gene can be the same as those of the ES cells. As we discuss here, differences in genetic background have been shown to significantly impact tumour susceptibility, which can lead to misinterpretation of results. This strain-dependent phenotypic variation can manifest as completely different phenotypes or differential penetrance. ${ }^{1,2}$

This issue of differential tumour susceptibility caused by the influence of genetic background is highlighted in a recent study by Biswas et al. ${ }^{3}$ published in Cell Death and Differentiation. The authors demonstrated that loss of Bid delays tumourigenesis in Atm-deficient mice by generating compound knockout mice $\left(\mathrm{Atm}^{-/-} \mathrm{Bid}^{-/-}\right)$. This was achieved by cross-breeding $\mathrm{Atm}^{+/-}$mice (129S/Sv background) to $\mathrm{Bid}^{-1-}$ mice (C57BL/6 background). Subsequently, tumour onset in $\mathrm{Atm}^{-1-} \mathrm{Bid}^{-1-}$ mice in a mixed genetic background (129S/Sv:C57BL/6) was compared to the $\mathrm{Atm}^{-1-}$ parental strain in a pure $129 \mathrm{~S} / \mathrm{Sv}$ background. Given the tumour suppressive influence of the C57BL/6 background, which renders this strain resistant to many types of tumours, ${ }^{1,4}$ the effects of Bid-deficiency on tumour susceptibility in this study are difficult to interpret.

In order to accurately compare congenic strains potentially confounding influence of mixed genetic backgrounds must be controlled for by ensuring all genotypes are comprised of the same genetic background. In the study by Biswas et al., ${ }^{3}$ the appropriate controls seem not included to definitively rule out the possibility that C57BL/6 background contributes to the delayed tumourigenesis observed in $\mathrm{Atm}^{-1-} \mathrm{Bid}^{-1-}$ mice.

Many studies employing GEM models have demonstrated how genetic background can cause substantial phenotypic variation. $^{1,2}$ A particularly striking example is the $N M Y C$ transgenic mouse model of neuroblastoma. Differential neuroblastoma penetrance between NMYC transgenic mice of different genetic backgrounds was reported. ${ }^{5}$ Serial backcrossing to a C57BL/6 background caused a reduced penetrance in contrast to serial backcrossing to a $129 \mathrm{~Sv} / \mathrm{J}$ background, which caused an increased penetrance.

This phenomenon has also been observed in other studies that compare tumour incidence in different genetic backgrounds. For example, tumour susceptibility in $p 53^{-1-}$ and $\mathrm{Atm}^{-1-}$ mice has also been shown to be dependent on genetic background. In a 129/Sv background, these knockout strains were found to have increased tumour penetrance compared to when they were backcrossed into a C57BL/6 background ${ }^{6,7}$.

Consistent with these observations, in our own experience in specific pathogen-free (SPF) conditions, we have observed that backcrossing once to a C57BL/6 background from a pure 129/Sv background significantly delays median tumour onset by $50-60 \%$ (Dorstyn et al., unpublished data). Taken together, these observations demonstrate the extent by which genetic background can impact on tumour susceptibility and highlights the importance of controlling for these effects.

Interestingly, multiple studies have provided evidence that environmental factors (for example, SPF versus non-SPF facilities, diet, and sanitation) are a major determinant of phenotypic variation between mouse models used in different studies. ${ }^{8,9}$ Although environmental factors no doubt influence tumour susceptibility (in addition to other phenotypes), it is highly unlikely that this completely explains the complex phenotypic variation observed in many studies. Rather, a combination of influences from environmental and genetic factors most likely explain these discrepancies.

Regardless of to what extent each of these factors contributes to phenotypic variation, suitable controls to rule out the effects of genetic background are essential for studies involving GEM models. Given the wealth of evidence supporting a role for genetic background in contributing to phenotypic variation, such studies that exclude these controls should be interpreted cautiously.

\section{Conflict of Interest}

The authors declare no conflict of interest.

$$
\begin{aligned}
& \text { J Puccini }{ }^{1,2}, \text { L Dorstyn }{ }^{1,2,3} \text { and S Kumar }{ }^{*, 1,2,3} \\
& 1 \text { Department of Haematology, Centre for Cancer Biology/SA Pathology, Frome } \\
& \text { Road, Adelaide, SA 5000, Australia; } \\
& 2 \text { Department of Medicine, University of Adelaide, Adelaide, SA 5005, Australia and } \\
& 3 \text { Division of Health Sciences, University of South Australia, Adelaide, SA 5001, } \\
& \text { Australia } \\
& \text { * Corresponding author: S Kumar, Department of Haematology, Centre for Cancer } \\
& \text { Biology, SA Pathology, PO Box 14, Rundle Mall, Adelaide, SA 5000, Australia. } \\
& \text { Tel: + 61 } 88222 \text { 3738; Fax: + 61 } 882223162 ; \\
& \text { E-mail: Sharad.Kumar@ health.sa.gov.au }
\end{aligned}
$$

1. Rivera J et al. Immunity 2008; 28: 1-4.

2. Doetschman T. Methods Mol Biol 2009; 530: 423-433.

3. Biswas S et al. Cell Death Differ 2013; 20: 869-877.

4. Klopstock N et al. PLoS One 2009; 4: e5025.

5. Weiss WA et al. EMBO J 1997; 16: 2985-2995.

6. Donehower LA et al. Mol Carcinog 1995; 14: 16-22.

7. Petiniot LK et al. Mol Cell Biol 2002; 22: 3174-3177.

8. Reliene R et al. DNA Repair 2006; 5: 651-653.

9. Valdar W et al. Genetics 2006; 174: 959-984. 